\title{
Pagamento por serviços ambientais em área de proteção aos mananciais: uma proposta para a região do ribeirão Balainho, Suzano, São Paulo
}

\author{
Joyce Marques Barbosa ${ }^{1}$ \\ Rafaela Quintanilha Abrahão ${ }^{1}$ \\ Claudete Bezerra dos Santos Canada ${ }^{1}$ \\ Carlos Alberto Mariotoni ${ }^{2}$ \\ ${ }^{1}$ Centro Universitário São Camilo \\ Rua Raul Pompéia, 144 - 05025-010 - São Paulo, SP, Brasil. \\ joycebarbosa.bio@gmail.com; rabiologa@gmail.com; claudete158@terra.com.br \\ ${ }^{2}$ Universidade Estadual de Campinas - UNICAMP \\ Faculdade de Engenharia Civil, Arquitetura e Urbanismo \\ Av. Albert Einstein, 951 - Caixa Postal: 6021- 13083-852 - Campinas, SP, Brasil. \\ cam@fec.unicamp.br
}

\begin{abstract}
The relationship between demand and hydro availability, jointly to the spring catchments degradation, must be faced as one of the main threats to the sustainability of São Paulo metropolitan region (RMSP). Urbanization advances on supplies systems, intensifying the shortage of these supplies and of the places for purification of water, as well as the lost of this quality, being necessary creating measures that guarantee conservation. The environmental services that can be provided by agriculture are several, confirming the importance of farmers as agents for spring catchments preservation. However, they do not have sustainable conditions to bear the countless pressures that surround them. This work aims to propose the implementation of payments for environmental services (PES) for the farmers in the Balainho's subcatchment region, in Suzano (SP) included in a spring catchments protection area, using the amount of charge for water use for the payment. For this purpose, a bibliographic research and a case study have been carried out. The environmental valuation and their methods support the insertion of environmental responsibilities within political and market mechanisms. In this context, the PES services represents a recent instrument, with emergency and inductive character, capable of providing the necessary stability for the farmers, enabling them to perform their multifunctional role. Despite the farmers' importance in the spring catchments protection, there are losses of these areas in Balainho region. In order to revert this situation, payments for environmental services are proposed, which will be measured using opportunity cost methods and/or reposition costs, and to guarantee the environmental consciousness, educational instruments will be used.
\end{abstract}

Palavras-chave: Incentives and Environmental Subsidies; protected water supplies; Environmental education; Incentivos e Subsídios Ambientais; Mananciais protegidos; Educação ambiental.

\section{Introdução}

Os mananciais se tornam cada vez mais estratégicos para a sobrevivência das cidades devido ao aumento acelerado da demanda e do comprometimento qualitativo da água destinada ao abastecimento das populações urbanas. Entretanto, ao mesmo tempo, a urbanização avança sobre esses espaços, destruindo suas condições de produzir e depurar a água. Na Região Metropolitana de São Paulo (RMSP), essa situação deve ser encarada como uma das principais ameaça a sustentabilidade (TAGNIN; MAGALHÃES, 2001; WHATELY; TAGNIN, 2006). Em um primeiro momento, quando se fala da problemática enfrentada nas regiões de mananciais da RMSP, logo vem em mente à representação de paisagens com intensa ocupação urbana desordenada e poluição das águas, como ocorre nas represas Billings e Guarapiranga. Nesse contexto, a situação é tratada por meio de medidas com o objetivo de corrigir os impactos já causados, ou seja, são tratadas por intermédio das chamadas Soluções Ambientais. Porém a 
RMSP possui também outras áreas de mananciais mais preservadas, que, apesar de serem de grande importância, acabam recebendo menos atenção do que o merecido, talvez pelo fato de apresentarem uma situação menos emergencial (AQUINO, 2008; ISA, 2008).

Esse é o caso da sub-Bacia do Alto Tiete Cabeceiras, pois parte considerável dela ainda preserva características de baixa densidade populacional. Dessa forma, seu maior desafio de gestão é identificar instrumentos que garantam a preservação da área, assegurando a manutenção e podendo até ampliar a capacidade de produção de água para o abastecimento da região metropolitana, e ao mesmo tempo não prejudicando o desenvolvimento econômico da região (CARVALHO; FRANCA, 2005).

Um manejo adequado requer investimento e, todavia, na maior parte dos casos é inviável no âmbito econômico, principalmente para agricultores de médio e pequeno porte. Nesse contexto faz-se presente a possibilidade de implementação de pagamento por serviços ambientais. Assim, uma vez que o agricultor vise à preservação dos mananciais, ele tem o direito de receber pagamento pelo serviço ambiental prestado. Dessa forma, poderá investir cada vez mais recursos na preservação e se desenvolver de forma mais efetiva, melhorando também sua qualidade de vida. O presente artigo tem como objetivo a criação de uma proposta de implementação de pagamentos por serviços ambientais na região da microbacia do ribeirão Balainho, inserida no município de Suzano - SP, e ainda, a utilização do montante obtido pela cobrança de uso da água para o referido pagamento e a ferramenta da educação ambiental como mecanismo de conscientização aos agricultores da região garantindo a sustentabilidade dos mananciais.

\section{Materiais e Métodos}

O estudo teve como foco a microbacia do ribeirão Balainho pertencente ao distrito de Palmeiras, inserida no município de Suzano - SP (Fig 1.), localizado completamente em área de proteção aos mananciais, sendo os setores de maior densidade os bairros: Recanto Feliz, Jardim Belém, Vila Real Palmeiras, Jardim Itamaracá, com densidades entre cinqüenta e cem habitantes/hectares (SUZANO, 2007).

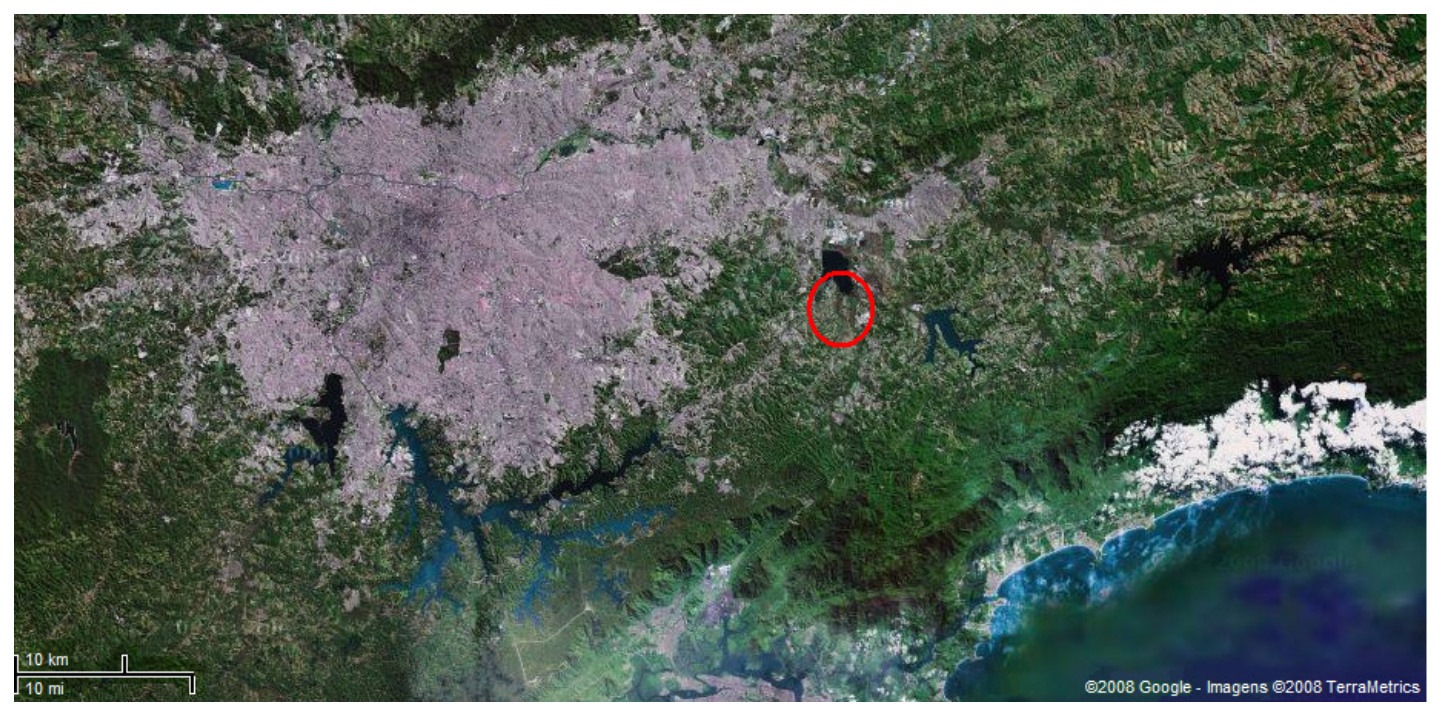

Figura 1. Vista da mancha urbana da Região Metropolitana de São Paulo e seus arredores, indicando a área onde está inserida a microbacia do ribeirão Balainho (em vermelho) (GOOGLE MAPS, 2008). 
A região do balainho pertencente a sub-bacia alto Tietê cabeceiras, localizado a leste da cidade de São Paulo. A microbacia do ribeirão Balainho possui uma área total de 3566,6 ha, um perímetro de $43816,4 \mathrm{~m}^{2}$ e é caracterizada por ser uma área de declive em sua parte alta e relevo plano com suaves ondulações em sua parte baixa, sendo por esse motivo dividido entre baixo, médio e alto balainho (fig. 2) (VICENTE et al., 2006; AQUINO, 2008). O Balainho possui uma malha hidrológica muito rica, com 64 quilômetros de rios e 147 nascentes. Da área total da região do Balainho, em torno de $20 \%$ se encontra em Área de Proteção Permanente (APP), o que correspondendo a 723 ha. (AQUINO, 2008). Segundo Teixeira (2008) a microbacia do ribeirão Balainho é enquadrada na área de $2^{\mathrm{a}}$ categoria dentro da Lei $1.172 / 76$. Sua área é predominantemente classificada como classe C. Somente não é classificada como tal o Bairro da V-Divisão, classificada como classe A, abrangendo uma área de 4,4 hectares.

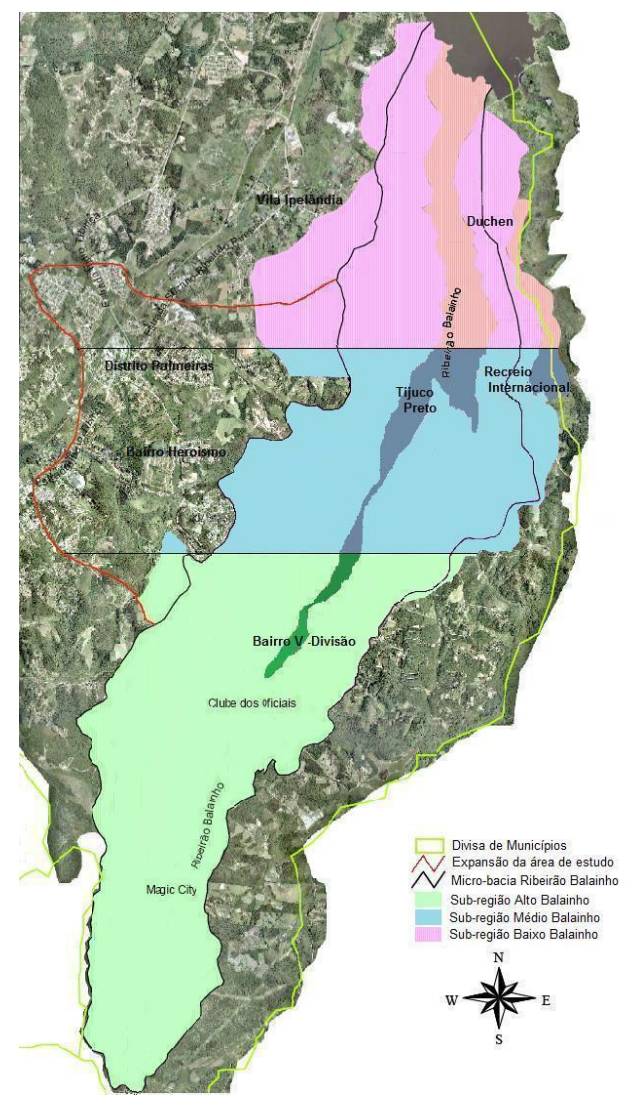

Figura 2. Delimitação da área de estudo da microbacia do ribeirão Balainho, redesenhada (em vermelho) para incluir áreas limítrofes que se integram à vida social da população (Modificado FERREIRA, 2004).

A região da microbacia do ribeirão Balainho é a área de maior importância agrícola de Suzano e uma das regiões de mananciais que ainda se encontra protegida. Entretanto, a expansão da mancha urbana nessa região é evidente.

De acordo com Moraes e Carvalho ([21-]) entre o ano de 1972 e 2002, houve grande expansão das áreas urbanas na região do balainho, passando de 16,5 ha para 244 ha, assim como grande expansão de áreas de loteamento, tendo sido constatado um aumento de $89 \%$. O avanço da mancha urbana é responsável por acentuar os problemas de infra-estrutura já existentes, em especial o serviço de coleta de esgoto. Essa defasagem no serviço de saneamento é problema 
tanto nas áreas urbanas quanto nas rurais da região do Balainho, o que agrava os problemas ambientais e degrada os mananciais.

A perspectiva das famílias se manterem na região do balainho é de aproximadamente $87 \%$, entretanto, somente $40 \%$ delas pretendem se manter na atividade agrícola (FERREIRA, 2004; CARVALHO et al., 2006). Esse fato se deve em parte a própria lei de proteção aos mananciais, que exige manejo adequado sem garantir subsídios para tal.

Segundo levantamentos de Antoniazzi et al. ([21-]) na região do Balainho, os tipos de agricultura encontrados são: horticultura convencional e horticultura de monocultura, ambos são considerados sistemas agrícolas insustentáveis, não se adequando a região de mananciais. Porém, a lei 1.172/76 restringe o uso do solo e torna necessária a adoção de métodos mais adequados. A agricultura orgânica é considerada adequada para a região, entretanto parece se inviabilizar economicamente para os produtores, por resultar em uma rentabilidade negativa (NOSSE et al., [21-]).

O estudo de caso foi realizado a partir observação simples e análise documental da regiãoalvo, na microbacia do ribeirão Balainho, no município de Suzano (SP), localizado a leste da cidade de São Paulo, na latitude S $23^{\circ} 31^{\prime} 20^{\prime \prime}$ e longitude W 46¹' $52^{\circ}$ '. Para definir a área a ser estudada no presente trabalho, foi tomada como base a delimitação proposta pelo projeto "Negociando Conflitos sobre a Água em Zonas Peri-urbanas"(NEGOWAT). Entretanto, neste trabalho a delimitação da microbacia do ribeirão Balainho foi redesenhada para incluir áreas limítrofes que se integram à vida social da população, incorporando áreas contíguas dos bairros rurais, áreas de produção e três áreas de concentração urbana: Vila Ipelândia, Bairro de Palmeiras e Região Recreio Internacional.

A visita exploratória foi realizada por intermédio de observação simples, acompanhada pelo supervisor da equipe do Alto Tietê do Departamento de Uso do Solo Metropolitano, para que fosse feito o reconhecimento da região, com o propósito de registrar, por meio de fotografias, suas principais atividades comerciais. A exploração foi feita com o auxílio de mapas topográficos. Para a análise documental foram utilizados documentos obtidos no sites de busca Google acadêmico e diretamente no Sindicato Rural de Mogi das Cruzes e na Casa da Agricultura de Suzano, com o uso das palavras-chave: município de Suzano e microbacia do ribeirão Balainho.

A proposta foi construída com base nos dados obtidos com o levantamento bibliográfico, principalmente quanto às experiências de pagamento por serviços ambientais apresentadas, no caso "Projeto Conservador das Águas" e "Projeto Oásis". A partir deles, procurou-se adequar o pagamento por serviços ambientais à realidade da região do Balainho, visualizada por meio do estudo de caso.

\section{Resultados e Discussão}

Por ser a principal atividade da microbacia do Ribeirão Balainho e ser potencialmente compatível com as exigências impostas em áreas de mananciais, a manutenção das áreas agrícolas é uma estratégia adequada para a preservação dos mananciais. A manutenção de áreas agrícolas, quando manejadas corretamente, além de prestar serviços ambientais diversos, como a conservação do solo e da água e manejo sustentável da biodiversidade, além de impedir a expansão urbana ou de outras atividades de impacto ambiental.

Com base na importância da agricultura na região do Ribeirão do Balainho, a função ambiental que os agricultores produzem para a sociedade, e a tendência de expulsão deles de suas 
terras devido sua instabilidade frente às crescentes pressões às quais estão sujeitos, pode-se afirmar que o pagamento por serviços ambientais é, além de justo, necessário.

Visando a proteção de mananciais da Região Metropolitana de São Paulo, a proposta elaborada caminha no sentido de tornar possível a sustentabilidade dos agricultores da microbacia do Ribeirão Balainho, transformando-os em prestadores de serviços ambientais.

A implementação de pagamento por serviços ambientais na microbacia do Ribeirão Balainho poderá ser dividida em quatro etapas: diagnóstico, planejamento, implantação e manutenção, sendo as duas últimas devidamente fiscalizadas (Fig. 3). A fiscalização é um fator chave para a efetividade da proposta.

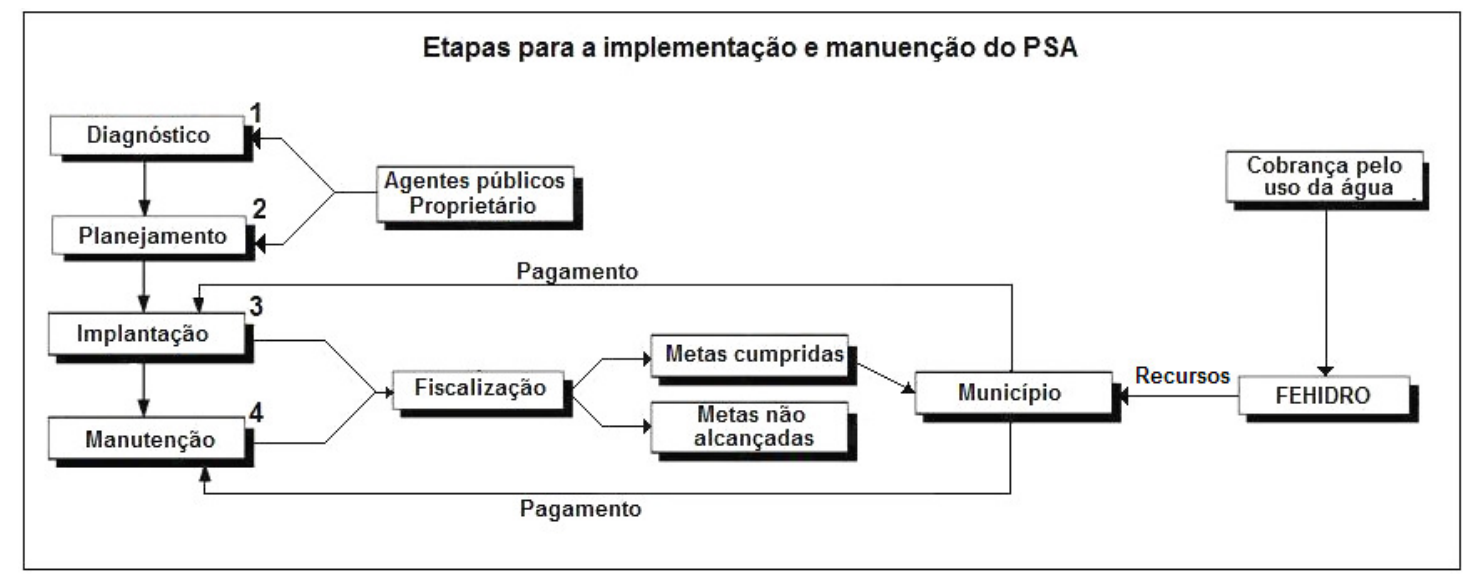

Figura 3. Etapas para a implementação e manutenção do PSA (Autoria própria, 2008).

$\mathrm{Na}$ etapa do diagnóstico deverá ser avaliada a situação de cada propriedade, a fím de verificar alguns aspectos importantes, entre eles o licenciamento da propriedade; o zoneamento do território, verificando a existência de áreas protegidas por lei, como no caso de APP; o sistema de produção agrícola e as externalidades geradas.

Em virtude do estudo de caso, pontos importantes a serem diagnosticados nas propriedades são a existência de serviços de coleta de lixo, esgoto e abastecimento de água, além do sistemas de irrigação e outras tecnologias que dizem respeito ao manejo dos recursos hídricos e do solo. Todos esses fatores devem ser adequados para garantir a preservação dos manancias.

O planejamento poderá ser realizado por um agente público em conjunto com o próprio produtor rural. A partir dos dados obtidos pelo diagnóstico, serão analisadas as melhores formas de adequação da propriedade e do modo de produção agrícola. Nessa fase está incluída a mensuração do valor do pagamento pelos serviços ambientais prestados pelo produtor e, ao fim desta, ocorrerá o fechamento do contrato por ambas as partes.

O pagamento apenas será efetuado caso os proprietários rurais cumpram as metas impostas pelo contrato. Os primeiros pagamentos serão necessariamente investidos para a concretização das metas presentes no contrato, ou seja, para possibilitar a etapa da implantação. Os proprietários só receberão o pagamento por serviços ambientais caso cumpram todas as exigências propostas e tenha sua propriedade licenciada, o que, além de importante para a preservação, auxilia no controle de registro das propriedades.

Por fim, após a etapa de implantação, com a devida fiscalização, o produtor começará a receber o pagamento por serviços ambientais propriamente ditos. Entretanto para a etapa de manutenção, na qual as atividades da propriedade serão fiscalizadas de forma periódica, se as 
metas estiverem sendo cumpridas de forma adequada o produtor receberá a verba proveniente do PSA; caso suas metas não estejam sendo cumpridas, ele deixa de receber o pagamento. Nesse caso, depois de verificado as dificuldades para o cumprimento das metas, deverão ser realizadas um novo acordo, para que o agricultor volte a receber, caso cumpra as metas.

É proposto que o PSA seja um instrumento permanente, ou até que seja implementado outro instrumento que garanta a permanência dos agricultores ou garanta a conservação da região, e, consequentemente a qualidade da água.

Os recursos financeiros para o pagamento de serviços ambientais consistirão em parte do montante arrecadado com a lei da cobrança pelo uso da água, podendo-se obter outras formas de captar recursos. É proposto que o pagamento por serviços ambientais seja inserido como instrumento nas leis especificas da Bacia Hidrográfica do Alto Tietê cabeceiras, em busca da perpetuação do projeto para toda a região.

Outros casos de aplicação de PSA têm mostrado resultados positivos, porém ainda insustentável perante aos agricultores, segundo dados dos Dispersores (2007), já que os recursos financeiros destinados a esse fim limitam-se às parcerias. Por conta disso, sugere-se a inserção do PSA na Lei Específica do Alto Tietê Cabeceiras, a qual está atrelada à lei da cobrança de uso da água, garantindo assim maiores subsídios.

Para calcular os valores a serem pagos para cada propriedade, deverão ser adotados métodos de valoração ambiental. Pelo fato de os métodos diretos de valoração apresentarem subjetividade quanto à valoração dos serviços ambientais, acredita-se que o melhor caminho a seguir é a adoção de métodos indiretos. Partindo desse pressuposto e com base no observado por meio do estudo de caso, propõe-se os seguintes métodos de valoração ambiental: Custo de oportunidade e Custo de reposição.

O custo de oportunidades representa as perdas econômicas da população em virtude das restrições de uso dos recursos ambientais. Conforme Dernadin (2004) o custo de oportunidades deveria ser feito a partir da contabilização da máxima exploração que o proprietário de terra poderia ter em cima dessas. Entretanto propomos que a estimação monetária do aproveitamento da terra deve ser restritiva, podendo ser valorado somente atividades sustentáveis, sendo assim custo de oportunidade ambiental.

Ou, ainda, se um produtor agrícola possuir em seu terreno uma área de nascente, será proposto a ele que isole a mesma, com o propósito de protegê-la. No caso de essa área apresentar degradação, além de isolá-la, o produtor terá o compromisso de restaurá-la. Todo o custo gerado por essas ações será financiado por meio de pagamento por serviços ambientais. Além disso, caso a área isolada implique perda econômica para o produtor, como, por exemplo, a perda de espaço para sua produção, e em virtude disso ocorrer perda de renda, haverá a reposição do pagamento.

Para essa valoração propõe-se o seguinte cálculo baseado do trabalho realizado por Oliveira e Hoeflick (2007):

$$
\text { Valor da produção/ha }=\frac{\text { valor da produção }}{\text { área da produção }}
$$

Desta forma, é o valor da produção por hectare, e será pago ao agricultor em função de quantos hectares de produção ele perdeu por conta da preservação. No caso de uma área de preservação ambiental que não represente perda econômica para o agricultor, como no caso de uma área preservada, o método de valoração utilizado proposto é o de custo de reposição, que 
estima o custo para repor ou restaurar o recurso ambiental danificado. Por não depender de um trabalho de campo amplo, é um método de fácil aplicação.

Além disso, é proposto um projeto de educação ambiental juntamente a aplicação de pagamentos por serviços ambientais para subsidiar, por meio de capacitação em novas práticas de sustentabilidade, os agricultores nessa nova etapa. Para tal acredita-se na importância de receber instruções para tomar conhecimento das mudanças criadas pelo PSA, através de oficinas para promover a capacitação técnica para implantar um manejo adequado nas propriedades.

\section{Conclusão}

A proposta do PSA, ao garantir a sustentabilidade dos agricultores, aumenta sua perspectiva de permanência e permite implantar práticas adequadas de manejo e saneamento básico nas áreas de produção agrícola, abrangendo a maior parte dos fatores necessários para promover a proteção efetiva das áreas de mananciais.

Os métodos propostos de valoração ambiental: custo de oportunidade e custo de reposição, ambos são métodos adequados que calculam valores viáveis e consistentes que levam em consideração a necessidade de cada propriedade, proporcionando um pagamento que sustente os gastos obtidos com a conservação, ou seja, proporcionando a sustentabilidade dos agricultores. Além disso, esses consistem em métodos complementares, com capacidade de abranger a maior parte de situações possíveis, são de fácil aplicabilidade, sendo os seus cálculos simples, que não necessitam de estudos amplos.

\section{Referências}

ANTONIAZZI, L. B. et al. Custo de Produção e Rentabilidade de Subsistemas de Produção, na Região de Cabeceiras do Alto Tietê, [S.I], [21-], p 1-15.

AQUINO, B. M. Comunicação oral. Casa de agricultura de Suzano. Entrevista. Out. 2008.

CARVALHO, Y. M. C.; FRANCA, T. J. F. A preservação dos mananciais da Região Metropolitana de São Paulo e a multifuncionalidade, jan. 2005. IEA APTA. 16 p. Informe de trabalho Negowat Brasil No 17. Disponível em:

<http://www.icarrd.org/en/proposals/Multifunc_rural.pdf>. Acesso em: 19 out. 2008.

CARVALHO, Y. M. C. Agricultura: serviço ambiental para a Bacia do Alto Tietê-

Cabeceiras. São Paulo em Perspectiva, São Paulo, v. 20, n. 2, p.118-135, jun. 2006.

DENARDIN, Anderson Antonio. A importância do custo de oportunidade para a avaliação de empreendimentos baseados na criação de valor econômico (Economic vale added EVA). Contexto, Porto Alegre, v. 4, n. 6, p.1-19, 2004

DISPERSORES (Ed.). Visita ao Projeto Conservador das Águas no município de Extrema. Disponível em: <http://www.dispersores.org/atividades.php?id=22>. Acesso em: 4 set. 2008.

FERREIRA, S. E. Caracterização do sistema agrário da Micro-Bacia Hidrográfica do Ribeirão Balainho, pertencente à sub-bacia hidrográfica Alto Tietê-Cabeceira, Município de Suzano, 2004. Mimeo. 
GOOGLEMAPS. Vista da mancha urbana da Região Metropolitana de Paulo e seus arredores, indicando (em vermelho) a área onde está inserida a microbacia do ribeirão Balainho. Disponível em: <maps.google.com.br>. Acesso em 25 set., 2008.

ISA - INSTITUTO SOCIOAMBIENTAL. Mananciais da Região Metropolitana de São Paulo. 2005. Disponível em: <http://www.socioambiental.org/prg/man.shtm>. Acesso em: 19 abr. 2008.

MORAES, J F L de; CARVALHO, J P de. Caracterização e Evolução do Uso das Terras nas Microbacias do Córrego Balainho e Parelheiros. Serviço Ambiental da Agricultura: Alto Tietê - Região Metropolitana de São Paulo, [s.i], n. , p.1-11, [21-].

NOSSE, T O; ANTONIAZZI, L B. Custo de Produção e Rentabilidade de Subsistemas de Produção, na região de Cabeceiras do Alto Tiête. Serviço Ambiental da Agricultura: Alto Tietê - Região Metropolitana de São Paulo, [s.i], n. , p.1-15, [21-].

OLIVEIRA, S. O; HOEFLICK. V. A. análise do custo de oportunidade para conservação da floresta de araucária no estado do Paraná. IX ENGEMA - Encontro Nacional sobre Gestão Empresarial e Meio Ambiente. Curitiba, 19 a 21 de novembro de 2007. Disponível em: <http://engema.up.edu.br/arquivos/engema/pdf/PAP0034.pdf>. Acesso em: 3 nov. 2008.

SUZANO (MUNICÍPIO). Prefeitura Municipal de Suzano. Secretaria de Política Urbana. Revisão do Plano Diretor de Suzano 2006-2016: Etapa 4 - Construção do Conhecimento sobre a Realidade Local. jan. 2007.

TAGNIN, Renato Arnaldo; MAGALHÃES, Edmundo de Werna. O tratamento da expansão urbana na proteção aos mananciais: o caso da Região Metropolitana de São Paulo. São Paulo: Boletim Técnico da Escola Politécnica da Usp, Departamento de Engenharia de Construção Civil, 2001. 28 p.

TEIXEIRA. J. A. G. Comunicação Oral. Região do Balainho.Casa de agricultura de Mogi das Cruzes. 2008.

VICENTE, M. C. M et al. Representatividade da tipologia de sistemas agrários nas subbacias Tietê-Cabeceiras e Guarapiranga e nas micro bacias de Balainho e Parelheiros, em São Paulo, Brasil. In: Artigos APTA-NEGOWAT. São Paulo, APTA, 2006.

WHATELY, Marussia; TAGNIN, Renato. Por um olhar metropolitano para garantir a sustentabilidade dos mananciais. 2006. Disponível em:

$<$ http://www.mananciais.org.br/site/mergulhe_nessa/artigos/porumolharmetropolitano $>$. Acesso em: 20 set. 08. 